\title{
Freeze-out parameters: lattice meets experiment
}

\author{
S. Borsanyi ${ }^{1}$, Z. Fodor ${ }^{1,2,3}$, S. D. Katz ${ }^{2.4}$, S. Krieg ${ }^{1,3}$, C. Ratti ${ }^{5}$, K. K. Szabo ${ }^{1}$ \\ 1 Department of Physics, Wuppertal University, \\ Gaussstr. 20, D-42119 Wuppertal, Germany \\ 2 Inst. for Theoretical Physics, Eötvös University, \\ Pázmány P. sétány 1/A,H-1117 Budapest, Hungary \\ 3 Jülich Supercomputing Centre, \\ Forschungszentrum Jülich, D-52425 Jülich, Germany \\ 4 MTA-ELTE "Lendület" Lattice Gauge Theory Research Group, \\ Pázmány P. sétány 1/A, H-1117 Budapest, Hungary \\ 5 Dip. di Fisica, Università di Torino and INFN, Sezione di Torino \\ via Giuria 1, I-10125 Torino, Italy
}

(Dated: May 23, 2013)

\begin{abstract}
We present our results for ratios of higher order fluctuations of electric charge as functions of the temperature. These results are obtained in a system of $2+1$ quark flavors at physical quark masses and continuum extrapolated. We compare them to preliminary data on higher order moments of the net electric charge distribution from the STAR collaboration. This allows us to determine the freeze-out temperature and chemical potential from first principles. We also show continuumextrapolated results for ratios of higher order fluctuations of baryon number. These will allow to test the consistency of the approach, by comparing them to the corresponding experimental data (once they become available) and thus extracting the freeze-out parameters in an independent way.
\end{abstract}

The QCD transition from a hadronic, confined system to a partonic one at zero baryo-chemical potential is an analytic cross-over, as was unambiguously shown by lattice QCD simulations [1]. This feature extends to small chemical potentials covered by the high energy runs at RHIC. The possibility that the transition becomes first order at large chemical potentials has triggered the low energy runs at RHIC, soon to be followed by the CBM experiment at the GSI, in search for the elusive critical point. In order to successfully spot its position, one needs to define observables which are sensitive to the change in the order of the phase transition. Event-byevent higher order fluctuations of conserved charges are expected to diverge in the presence of a first order phase transition, and have therefore been proposed long ago to this purpose [2 4]. As a consequence, experimental results for these observables are becoming available at several collision energies, covering different regions of the QCD phase diagram [ $[$,, 6$]$. Recently, further interest towards fluctuations of conserved charges and their ratios has been stimulated even at $\mu=0$, following the idea that the freeze-out parameters can be extracted by comparing their experimental value to lattice QCD results [7, 8]. This comparison allows to extract the temperature and baryon-chemical potential at freeze-out from first principles, without the need of relying on a phenomenological model such as the Hadron Resonance Gas (HRG). This also allows to test the assumption that the equilibrium system simulated on the lattice is suitable to describe the experimentally measured fluctuations, since in principle non-equilibrium effects and final-state interactions in the hadronic phase might become relevant. The present level of precision reached by lattice
QCD simulations, performed at physical quark masses and continuum-extrapolated, is very timely and allows this kind of comparison between experimental data and lattice QCD results for the first time.

In this paper we show the first continuum-extrapolated results for higher order fluctuations of electric charge and extract the freeze-out conditions by comparing our results to preliminary data by the STAR collaboration at RHIC [5, 6]. This follows our previous work on secondorder fluctuations of conserved charges [9]. We also present results for baryon number fluctuations, which can be compared to the experimental data, once they become available (so far, only proton fluctuations have been measured in experiments [10], and the issue whether one can extract baryon number fluctuations from them is still open [11, 12]). Our simulations are performed in a system of $2+1$ quark flavors at the physical point, i.e. with physical $M_{K} / f_{K}$ and $M_{\pi} / f_{K}$ ratios at each lattice spacing, which are realized at the strange- over light-quark mass ratio $m_{s} / m_{u, d} \simeq 28$.

The continuum extrapolation is mainly performed on the basis of five lattice spacings, corresponding to temporal lattice extents of $N_{t}=6,8,10,12,16$ (around $T_{c}$ these extents result in lattice spacings of $a=0.22,0.16,0.13,0.11$ and $0.08 \mathrm{fm}$, respectively). At every lattice spacing and temperature we analyzed every 10th configuration in the rational hybrid Monte Carlo streams with $128 \ldots 256$ quartets of random sources. The statistics for each point is shown in Fig. 11 We follow the extrapolation strategy that we have discussed in Ref. 9], and perform several possible continuum fits (with and without a beyond- $a^{2}$ term, keeping or dropping the coarsest lattice, using tree-level improvement [13] or 


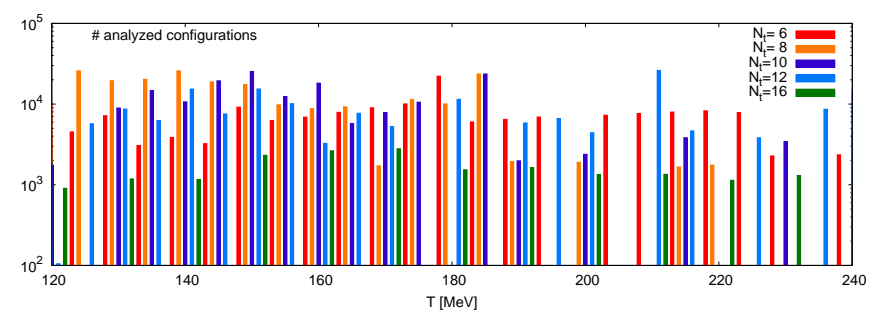

FIG. 1. Number of analyzed configurations for each temperature and each lattice spacing. The configurations have been saved with a separation of 10 trajectories. Each configuration was analyzed by $(128 \ldots 256) \times 4$ random sources.

not, fitting the observable or the reciprocal of the observable, choosing between two possible interpolations). Weighting these continuum results by the goodness of the fit a histogram is formed, the width of which defines the systematic error (for details see Ref. [14]). In this paper we show the combined systematic and statistical errors on the continuum data.

Similarly to previous works, we choose a tree-level Symanzik improved gauge, and a stout-improved staggered fermionic action (see Ref. [15] for details). The stout-smearing [16] reduces taste violation (this kind of smearing has one of the smallest taste violations among the ones used in the literature for large scale thermodynamic simulations, together with the HISQ action [17, 18] used by the hotQCD collaboration). This lattice artifact needs to be kept under control when studying higher order fluctuations of electric charge, which are piondominated at small temperatures, and thus particularly sensitive to this issue.

The observables under study are defined as:

$$
\frac{\chi_{l m n}^{B S Q}}{T^{l+m+n}}=\frac{\partial^{l+m+n}\left(p / T^{4}\right)}{\partial\left(\mu_{B} / T\right)^{l} \partial\left(\mu_{S} / T\right)^{m} \partial\left(\mu_{Q} / T\right)^{n}} .
$$

and they are related to the moments of the distributions of the corresponding conserved charges by

$$
\begin{aligned}
\text { mean : } M=\chi_{1} & \text { variance : } \sigma^{2}=\chi_{2} \\
\text { skewness : } S=\chi_{3} / \chi_{2}^{3 / 2} & \text { kurtosis : } \kappa=\chi_{4} / \chi_{2}^{2} .
\end{aligned}
$$

With these moments we can express the volume independent ratios

$$
\begin{aligned}
S \sigma & =\chi_{3} / \chi_{2} \quad ; \quad \kappa \sigma^{2}=\chi_{4} / \chi_{2} \\
M / \sigma^{2} & =\chi_{1} / \chi_{2} \quad ; \quad S \sigma^{3} / M=\chi_{3} / \chi_{1} .
\end{aligned}
$$

The experimental conditions are such, that the three chemical potentials $\mu_{B}, \mu_{Q}$ and $\mu_{S}$ are not independent of each other: the finite baryon density in the system is generated by the nucleon stopping in the collision region, and is therefore due to light quarks only. Strangeness conservation then implies that the strangeness density $\left\langle n_{S}\right\rangle=0$. Similarly, the initial isospin asymmetry of the colliding nuclei yields a relationship between the electric charge and baryon-number densities: $\left\langle n_{Q}\right\rangle=Z / A\left\langle n_{B}\right\rangle$. For $\mathrm{Au}-\mathrm{Au}$ and $\mathrm{Pb}-\mathrm{Pb}$ collisions, a good approximation is to assume $Z / A=0.4$.

Therefore, the dependence of $\mu_{Q}$ and $\mu_{S}$ on $\mu_{B}$ needs to be defined so that these conditions are satisfied. We take care of this by Taylor-expanding the densities with respect to the three chemical potentials up to order $\mu_{B}^{3}$ [8]:

$$
\begin{aligned}
& \mu_{Q}\left(T, \mu_{B}\right)=q_{1}(T) \mu_{B}+q_{3}(T) \mu_{B}^{3}+\ldots \\
& \mu_{S}\left(T, \mu_{B}\right)=s_{1}(T) \mu_{B}+s_{3}(T) \mu_{B}^{3}+\ldots
\end{aligned}
$$

These equations define $q_{1}, q_{3}$ and $s_{1}$ and $s_{3}$, respectively. Our continuum extrapolated data for the functions $q_{1}(T), q_{3}(T), s_{1}(T), s_{3}(T)$ are shown in Fig. 2. Our data are compared to the BNL-Bielefeld group's result, where $q_{1}$ and $s_{1}$ was continuum extrapolated. They obtained $q_{3}$ and $s_{3}$ from $N_{t}=8$ lattices using the HISQ action [8].
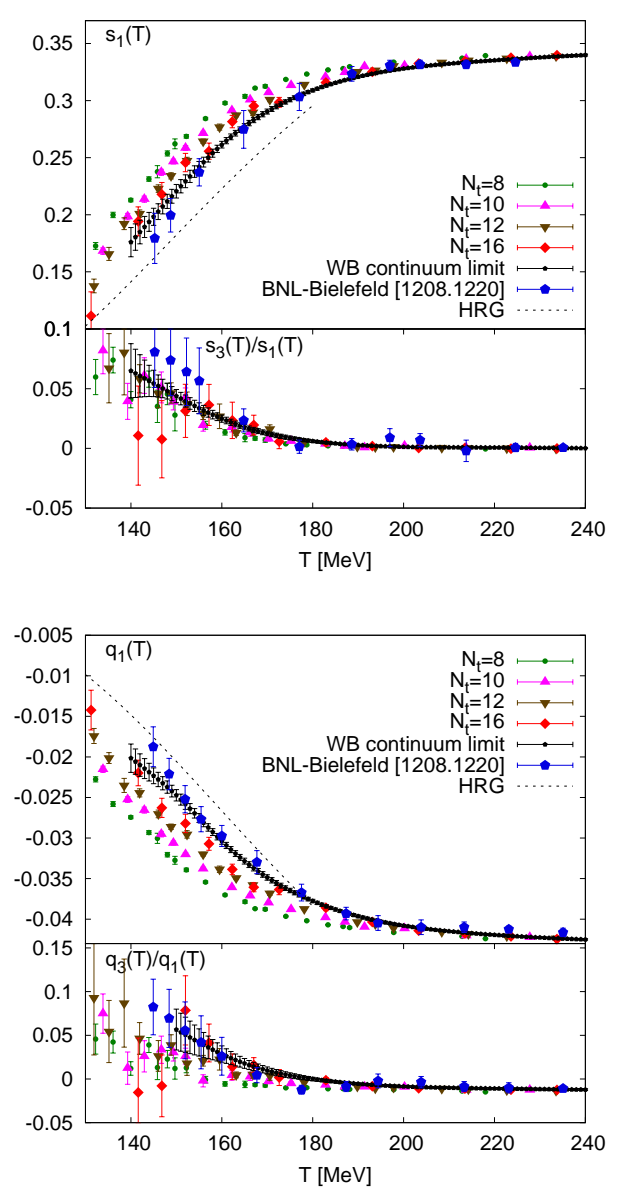

FIG. 2. Upper panels: leading order contribution in $\mu_{B}$ for the strangeness (upper figure) and the electric charge (lower figure) chemical potentials. The lower panels show the corresponding NLO contributions. In all panels, the black dots correspond to the continuum extrapolated results. The BNLBielefeld results are shown as blue pentagons. 


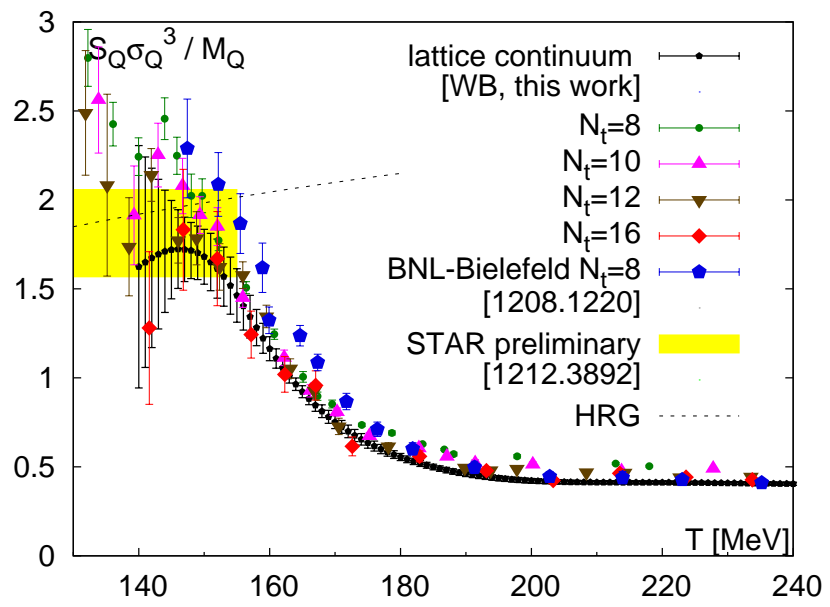

FIG. 3. $\quad R_{31}^{Q}$ : the colored symbols correspond to lattice QCD simulations at finite- $N_{t}$. Black points correspond to the continuum extrapolation; blue pentagons are the $N_{t}=8$ results from the BNL-Bielefeld collaboration [8]. The yellow band is the preliminary STAR measurement of $S_{Q} \sigma_{Q}^{3} / M_{Q}$ [6]: it has been obtained by averaging the two most central measurements from STAR over three collision energies: $\sqrt{s}=$ $27,39,62.4 \mathrm{GeV}$.

The quantities that we look at, in order to extract the freeze-out temperature and baryon chemical potential, are the ratios $\chi_{3}^{Q} / \chi_{1}^{Q}$ and $\chi_{1}^{Q} / \chi_{2}^{Q}$ at some $\left(\mu_{B}, \mu_{Q}, \mu_{S}\right)$ point, which is defined by the pyhsical conditions discussed in the previous paragraph and given by Eq. (4). We look at ratios because they are volume-independent, and also because they are directly related to the moments of charge distribution by Eqs. (3). The first terms of their Taylor expansion around $\mu_{B}=0$ read:

$$
\begin{aligned}
& R_{31}^{Q}\left(T, \mu_{B}\right)=\frac{\chi_{3}^{Q}\left(T, \mu_{B}\right)}{\chi_{1}^{Q}\left(T, \mu_{B}\right)}= \\
& \frac{\chi_{31}^{Q B}(T, 0)+\chi_{4}^{Q}(T, 0) q_{1}(T)+\chi_{31}^{Q S}(T, 0) s_{1}(T)}{\chi_{11}^{Q B}(T, 0)+\chi_{2}^{Q}(T, 0) q_{1}(T)+\chi_{11}^{Q S}(T, 0) s_{1}(T)}+\mathcal{O}\left(\mu_{B}^{2}\right)
\end{aligned}
$$

$R_{12}^{Q}\left(T, \mu_{B}\right)=\frac{\chi_{1}^{Q}\left(T, \mu_{B}\right)}{\chi_{2}^{Q}\left(T, \mu_{B}\right)}=$

$\frac{\chi_{11}^{Q B}(T, 0)+\chi_{2}^{Q}(T, 0) q_{1}(T)+\chi_{11}^{Q S}(T, 0) s_{1}(T)}{\chi_{2}^{Q}(T, 0)} \frac{\mu_{B}}{T}+\mathcal{O}\left(\mu_{B}^{3}\right)$.

The leading order in $\chi_{3}^{Q} / \chi_{1}^{Q}$ is independent of $\mu_{B}$, which allows us to use $R_{31}^{Q}$ to extract the freeze-out temperature. Once $T_{f}$ has been obtained with this method, the ratio $R_{12}^{Q}$ can then be used to determine $\mu_{B}$. Notice that in Eq. (5) we write the expansion of $R_{12}^{Q}$, but in the plots we will show our results up to NLO.

In Fig. 3 we show the ratio $R_{31}^{Q}$ as a function of the temperature. The continuum extrapolation, shown in the figure as black dots, is performed on the basis of five

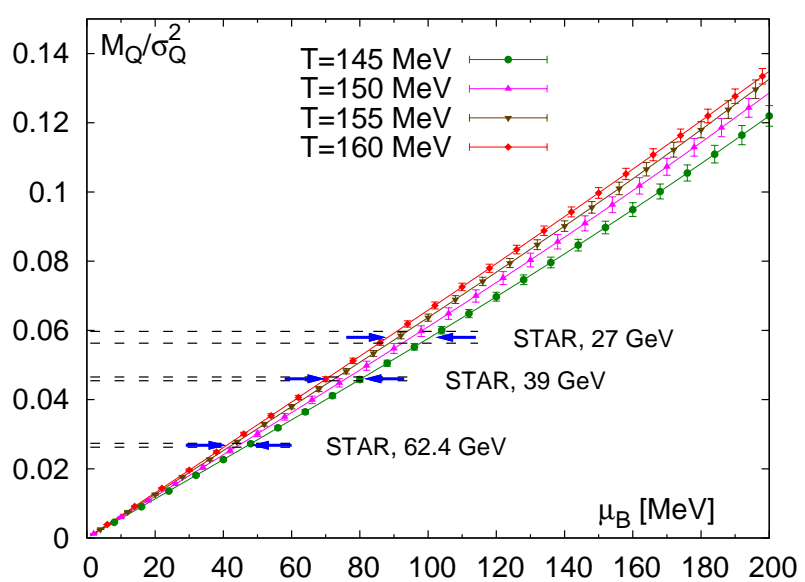

FIG. 4. $R_{12}^{Q}$ as a function of $\mu_{B}$ : the different colors correspond to the continuum extrapolated lattice QCD results, calculated at different temperatures. The three points correspond to preliminary STAR data for $M_{Q} / \sigma_{Q}^{2}$ at different collision energies: $\sqrt{s}=27,39,62.4$, from Ref. [6].

lattice spacings. Results from the BNL-Bielefeld collaboration corresponding to $N_{t}=8$ (from Ref. [8]) are also shown for comparison. The yellow band indicates the experimental value for $R_{31}^{Q}$ from the STAR collaboration 6]. It has been obtained by averaging the two most central measurements from STAR over three collision energies: $\sqrt{s}=27,39,62.4 \mathrm{GeV}$. We assume that this average safely allows to determine the freeze-out temperature, since the curvature of the phase diagram is very small around $\mu_{B}=0[19]$; therefore, we expect a small variation of $T_{f}$ over the chemical potential range corresponding to these three energies. Due to the big error-bar in the experimental measurement, and to the uncertainty in the lattice data at small temperatures, we can only get an upper limit for the freeze-out temperature: so far it appears that the freeze-out takes place at a temperature $T_{f} \lesssim 157 \mathrm{MeV}$. (Allowing for a two-sigma deviation both for the lattice simulation as well as the experimental data the highest possible freeze-out temperature is $161 \mathrm{MeV}$.)

In Fig. 4 we show our results for $R_{12}^{Q}$ as a function of the baryon chemical potential: the different curves correspond to different temperatures, in the range of $T_{f}$ determined from $R_{31}^{Q}$. The three STAR measurements, from Ref. [6], correspond to the collision energies $\sqrt{s}=$ $27,39,62.4$. Taking into account the limit on $T_{f}$ that we obtained through $R_{31}^{Q}$, the three values of $\mu_{B}$ that we extract from this observable are listed in Table \ The experimental evidence for the freeze-out temperature was just an upper bound (cf. Fig. 3), thus using the data in Fig. 4 can only provide for the $\mu_{B}$ prediction a lower bound. In Table 1 we assume that $T_{f}>145 \mathrm{MeV}$. The uncertainty in the freeze-out temperature is the dominant source of error. 


\begin{tabular}{|c|c|}
\hline$\sqrt{s}[\mathrm{GeV}]$ & $\mu_{B}^{f}[\mathrm{MeV}]$ \\
\hline 62.4 & $44(3)(1)(2)$ \\
39 & $75(5)(1)(2)$ \\
27 & $95(6)(1)(5)$ \\
& ()$_{\delta \mathrm{T}}()_{\text {lat }}()_{\exp }$ \\
\hline
\end{tabular}

TABLE I. Freeze-out baryon chemical potentials vs. the corresponding collision energy of the three STAR measurements from Ref. [6]. The errors come from the uncertainty of the freeze-out temperature, the lattice statistics and the experimental error, respectively. Notice that from Fig. 3 we were only able to obtain an upper limit on $T_{f}$. The values of $\mu_{B}$ and the error-bars in this table assume that $T_{f}$ is between 145 and $160 \mathrm{MeV}$, this uncertainty dominates the overall errors. (Doubling the experimental as well as lattice errors would increase full error only by a factor of 1.5.)

Note that these chemical potentials differ from the results of the statistical hadronization model [20, 21]. Also the typical freeze-out temperatures from the statistical fits lie above the upper bound found in this work.

In Fig. 5 we show our results for $R_{31}^{B}$ as a function of the temperature, while in Fig. 6 we show $R_{12}^{B}$ for different temperatures, as a function of $\mu_{B}$. Their Taylor expansions around $\mu_{B}=0$ read:

$$
\begin{aligned}
& R_{31}^{B}\left(T, \mu_{B}\right)=\frac{\chi_{3}^{B}\left(T, \mu_{B}\right)}{\chi_{1}^{B}\left(T, \mu_{B}\right)}= \\
& \frac{\chi_{4}^{B}(T, 0)+\chi_{31}^{B Q}(T, 0) q_{1}(T)+\chi_{31}^{B S}(T, 0) s_{1}(T)}{\chi_{2}^{B}(T, 0)+\chi_{11}^{B Q}(T, 0) q_{1}(T)+\chi_{11}^{B S}(T, 0) s_{1}(T)}+\mathcal{O}\left(\mu_{B}^{2}\right) \\
& R_{12}^{B}\left(T, \mu_{B}\right)=\frac{\chi_{1}^{B}\left(T, \mu_{B}\right)}{\chi_{2}^{B}\left(T, \mu_{B}\right)}= \\
& \frac{\chi_{2}^{B}(T, 0)+\chi_{11}^{B Q}(T, 0) q_{1}(T)+\chi_{11}^{B S}(T, 0) s_{1}(T)}{\chi_{2}^{B}(T, 0)} \frac{\mu_{B}}{T}+\mathcal{O}\left(\mu_{B}^{3}\right) .
\end{aligned}
$$

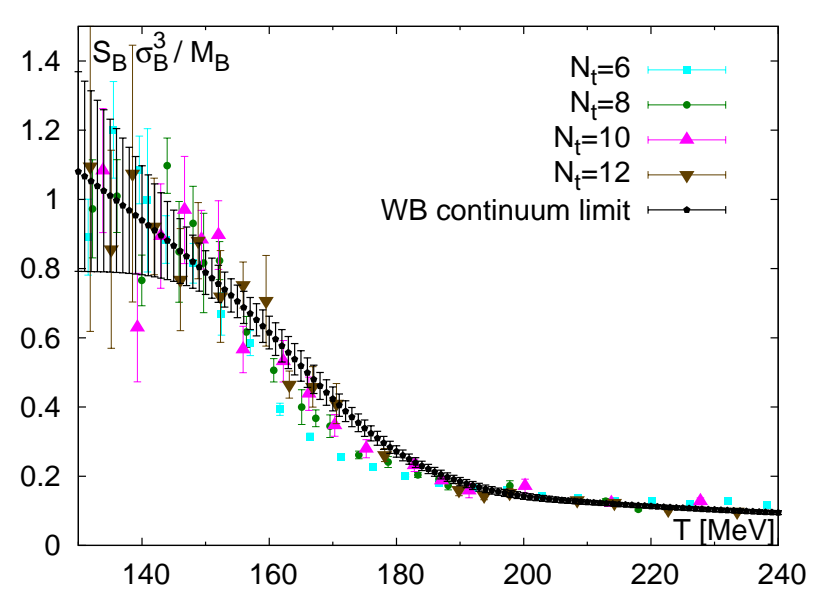

FIG. 5. $R_{31}^{B}$ : the colored symbols correspond to lattice QCD simulations at finite- $N_{t}$. The black points correspond to the continuum extrapolation.

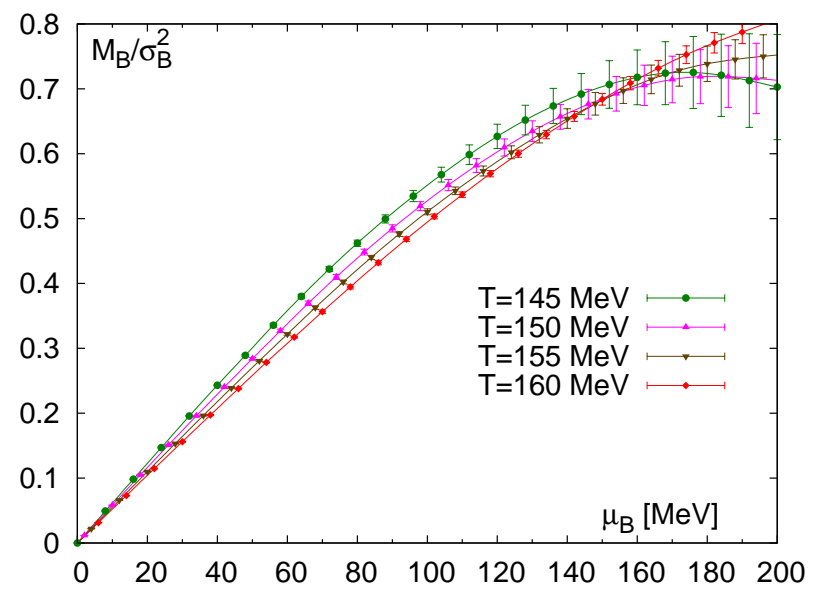

FIG. 6. $R_{12}^{B}$ : the colored symbols show the continuum extrapolated data at various temperatures. This quantity might also be used for $\mu_{B}$ measurement in the future.

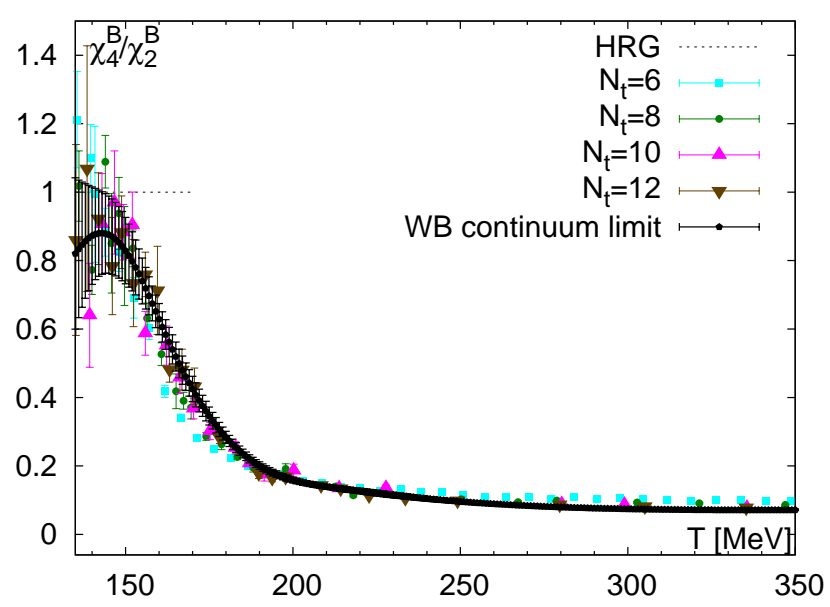

FIG. 7. $R_{42}^{B}$ as a function of the temperature. The black symbols correspond to the continuum extrapolation, the colored ones to the finite- $N_{t}$ simulations.

Therefore, similarly to the electric charge fluctuations, $R_{31}^{B}$ allows to extract $T_{f}$ and from $R_{12}^{B}$ we can then obtain $\mu_{B}$. This will allow to independently extract the freeze-out temperature and chemical potential by comparing them to the corresponding experimental values, once they become available. Notice that the ordering of the temperatures in Fig. 4 and Fig. [6] is opposite. $R_{12}^{B}$ might in future be used to set an upper bound for $\mu_{B}$. This cross-check is of fundamental importance: an inconsistency between the two sets of freeze-out parameters obtained from the electric charge and baryon number fluctuations might signal that it is not possible to treat the experimental system in terms of lattice QCD simulations in thermal equilibrium.

In Fig. 7 we show the ratio $R_{42}^{B}=$ $\chi_{4}^{B}\left(T, \mu_{B}\right) / \chi_{2}^{B}\left(T, \mu_{B}\right)$ as a function of the temper- 
ature. This observable corresponds to $\kappa \sigma^{2}$ of the baryon number distribution. It will allow to further independently extract $T_{f}$. Notice that, in the case of baryon number, the observables are essentially flat in the hadronic phase: if the experimental value should lie in the transition region $(T \gtrsim 150 \mathrm{MeV})$ we will be able to accurately determine $T_{f}$, if it lies in the hadronic phase we will only be able to provide an upper limit for the freeze-out temperature.

In conclusion, we have presented our continuumextrapolated results for ratios of higher-order fluctuations of electric charge and baryon number and compared them to recently measured moments of electric charge distribution from the STAR collaboration. This procedure has allowed us to extract, for the first time, the values for the freeze-out parameters $T_{f}$ and $\mu_{B}^{f}$ from first principles. So far it is only possible to extract an upper limit for $T_{f}$, due to both experimental and lattice QCD uncertainties. The value that we obtain, $T_{f} \lesssim 157 \mathrm{MeV}$, is well within the transition range predicted from lattice QCD simulations [22]. This is compatible with the expectation that freeze-out occurs just below the transition [23].

Acknowledgments: This project was funded by the DFG grant SFB/TR55. The work of C. Ratti is supported by funds provided by the Italian Ministry of Education, Universities and Research under the Firb Research Grant RBFR0814TT. S. D. Katz is funded by the ERC grant ((FP7/2007-2013)/ERC No 208740) as well as the "Lendület" program of the Hungarian Academy of Sciences ((LP2012-44/2012). The numerical simulations were performed on the QPACE machine, the GPU cluster at the Wuppertal University and on JUQUEEN (the Blue Gene/Q system of the Forschungszentrum Juelich).

[1] Y. Aoki, G. Endrodi, Z. Fodor, S. D. Katz and K. K. Szabo, Nature 443, 675 (2006) [hep-lat/0611014].

[2] M. A. Stephanov, K. Rajagopal and E. V. Shuryak, Phys.
Rev. D 60, 114028 (1999) [hep-ph/9903292].

[3] R. V. Gavai and S. Gupta, Phys. Rev. D 78, 114503 (2008) [arXiv:0806.2233 [hep-lat]].

[4] M. Cheng, et al., Phys. Rev. D 77, 014511 (2008) [arXiv:0710.0354 [hep-lat]].

[5] D. McDonald [STAR Coll.], arXiv:1210.7023 [nucl-ex].

[6] N. R. Sahoo [STAR Coll.], arXiv:1212.3892 [nucl-ex].

[7] F. Karsch, Central Eur. J. Phys. 10, 1234 (2012) [arXiv:1202.4173 [hep-lat]].

[8] A. Bazavov, et al., Phys. Rev. Lett. 109, 192302 (2012) [arXiv:1208.1220 [hep-lat]].

[9] S. Borsanyi, et al. JHEP 1201, 138 (2012) [arXiv:1112.4416 [hep-lat]].

[10] M. M. Aggarwal et al. [STAR Coll.], Phys. Rev. Lett. 105, 022302 (2010) [arXiv:1004.4959 [nucl-ex]].

[11] M. Kitazawa and M. Asakawa, Phys. Rev. C 86, 024904 (2012) [Erratum-ibid. C 86, 069902 (2012)] [arXiv:1205.3292 [nucl-th]].

[12] A. Bzdak, V. Koch and V. Skokov, Phys. Rev. C 87, 014901 (2013) [arXiv:1203.4529 [hep-ph]].

[13] S. Borsanyi, et al. JHEP $1011 \quad$ (2010) 077 [arXiv:1007.2580 [hep-lat]].

[14] S. Durr, Z. Fodor, J. Frison, C. Hoelbling, R. Hoffmann, S. D. Katz, S. Krieg and T. Kurth et al., Science 322 (2008) 1224 [arXiv:0906.3599 [hep-lat]].

[15] Y. Aoki, et al. JHEP 0601, 089 (2006)

[16] C. Morningstar and M. J. Peardon, Phys. Rev. D 69, 054501 (2004)

[17] A. Bazavov and P. Petreczky, PoS LAT2009, 163 (2009)

[18] A. Bazavov and P. Petreczky, arXiv:1005.1131 [hep-lat].

[19] G. Endrodi, Z. Fodor, S. D. Katz and K. K. Szabo, JHEP 1104, 001 (2011) [arXiv:1102.1356 [hep-lat]].

[20] A. Andronic, P. Braun-Munzinger and J. Stachel, Nucl. Phys. A 772 (2006) 167 [nucl-th/0511071] ; Phys. Lett. B 673 (2009) 142 [Erratum-ibid. B 678 (2009) 516] [arXiv:0812.1186 [nucl-th]].

[21] J. Cleymans, H. Oeschler, K. Redlich and S. Wheaton, Phys. Rev. C 73 (2006) 034905 [hep-ph/0511094].

[22] Y. Aoki, et al. Phys. Lett. B 643 (2006) 46; Y. Aoki, et al. JHEP 0906 (2009) 088; S. Borsanyi et al. [WuppertalBudapest Coll.], JHEP 1009, 073 (2010)

[23] P. Braun-Munzinger, J. Stachel and C. Wetterich, Phys. Lett. B 596 (2004) 61 